Applied Bioinformatics

\title{
RERconverge: an R package for associating evolutionary rates with convergent traits
}

\section{Amanda Kowalczyk ${ }^{1,2 *}$,Wynn K Meyer ${ }^{1 *}$,Raghavendran Partha ${ }^{1,2 *}$, Weiguang Mao ${ }^{1,2}$, Nathan L Clark ${ }^{1,2}$, Maria Chikina ${ }^{1,2 * *}$}

${ }^{1}$ Department of Computational and Systems Biology, University of Pittsburgh, Pittsburgh, PA, USA ${ }^{2}$ Joint Carnegie Mellon

University-University of Pittsburgh Ph.D. Program in Computational Biology, Pittsburgh, PA, USA

*Contribute equally to the work. ${ }^{* *}$ To whom correspondence should be addressed.

Associate Editor: XXXXXXX

Received on XXXXX; revised on XXXXX; accepted on XXXXX

\begin{abstract}
Motivation: When different lineages of organisms independently adapt to similar environments, selection often acts repeatedly upon the same genes, leading to signatures of convergent evolutionary rate shifts at these genes. With the increasing availability of genome sequences for organisms displaying a variety of convergent traits, the ability to identify genes with such convergent rate signatures would enable new insights into the molecular basis of these traits.

Results: Here we present the R package RERconverge, which tests for association between relative evolutionary rates of genes and the evolution of traits across a phylogeny. RERconverge can perform associations with binary and continuous traits, and it contains tools for visualization and enrichment analyses of association results.

Availability: RERconverge source code, documentation, and a detailed usage walk-through are freely available at https://github.com/nclark-lab/RERconverge Datasets for mammals, Drosophila, and yeast are available at https://bit.ly/2J2QBnj]

Contact:mchikina@pitt.edu

Supplementary information: Supplementary information, containing detailed vignettes for usage of RERconverge, are available at Bioinformatics online.
\end{abstract}

\section{Introduction}

A major motivation in evolutionary biology is to determine which genetic changes underlie phenotypic adaptations. Convergent evolution, in which the same phenotype arises independently in distinct evolutionary lineages, provides natural replicates of phenotypic adaptation that aid researchers in linking phenotypes to their underlying genetic changes. Selection repeatedly targets the same genes in several known cases of phenotypic convergence, including Prestin in echolocation in bats and marine mammals ( $\mathrm{Li}$ et al. 2010), $M c 1 r$ in reduced pigmentation in multiple vertebrate species (Kronforst et al. 2012), and $N a_{v} 1.4$ in toxin resistance in snakes (Feldman et al. 2012). This pattern leads to the prediction that genes responding selectively to a convergent environmental or phenotypic transition may show convergent shifts in evolutionary rates (i.e., number of nucleotide or amino acid substitutions per unit time), due to reduced or increased selective constraints on those genes. For example, both genes and non-coding elements involved in eye function lose selective constraint in subterranean mammals, and the corresponding rate shift signal can be used genome-wide to identify candidate regions with eye-specific function (Partha et al. 2017). Thus, evolutionary rates provide a rich source of genome-wide molecular data that, in combination with convergent phenotypes, can be used to infer targets of convergent selection.

Several research groups have developed methods to identify patterns of convergent evolutionary rate shifts associated with convergent changes in traits or environment, including Coevol (Lartillot and Poujol, 2011), Forward Genomics (Hiller et al. 2012. Prudent et al. 2016), and PhyloAcc (Hu et al. 2018). However, existing methods to identify these patterns still have 


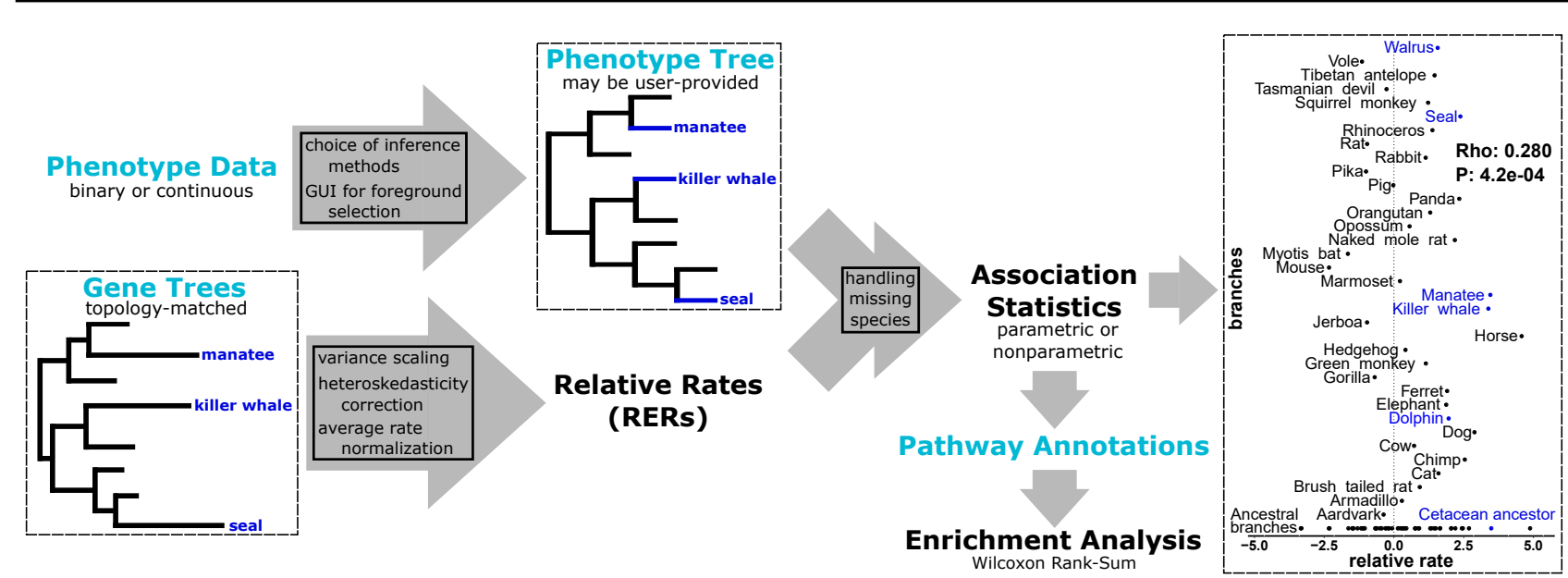

Fig. 1: Schematic of the RERconverge pipeline focusing on the example discussed in the case study. Far right figure shows RER for olfactory receptor OR9Q2, a top hit for marine-specific acceleration. For additional details of the methods (boxes in arrows), see (Partha et al. prep).

several areas for improvement, including support for both binary and continuous traits (Kowalczyk et al. prep), compute times that enable genome-wide analyses, robust statistical treatment (Partha et al. prep), and ease of visualization and downstream analyses. To address these issues, here we present RERconverge, a software package that implements tests of association between evolutionary rates of genetic loci and convergent phenotypic traits, both binary and continuous (pipeline illustrated in Figure 1). RERconverge incorporates and corrects for known phylogenetic relationships among included species, performs rapid analyses of genome-scale data and implements appropriate statistical techniques to reduce the effect of outliers and correct for multiple testing. This easy-toimplement software enables biologists with extensive organismal knowledge to apply computational techniques to quickly test hypotheses and link molecular data with phenotypes.

\section{Description and implementation}

\subsection{Basic usage of RERconverge}

The RERconverge package runs within an installation of the $\mathrm{R}$ software (R Core Team 2017) on any platform (Linux, Windows, Mac OS). The user provides the following data as input:

- A set of phylogenetic trees, all with the same topology, with branch lengths for each tree calculated from alignments of that "gene" sequence (these can also represent non-coding sequences such as enhancers) using software such as PAML (Yang 2007).

- Values for a trait of interest for extant species/tips of the tree. RERconverge can infer values for internal branches of the tree, or the user can provide these values via a single tree or by using RERconverge interactive branch selection to select foreground branches.

Detailed instructions for RERconverge are available within the Supplementary information to this article or at https://github.com/nclark-lab/RERconverge/wiki/Vignettes.

\subsection{Rapid estimation and visualization of Relative Evolutionary Rates (RER)}

RERconverge offers efficient computation of gene-specific rates of evolution on branches of phylogenetic trees in genomescale datasets. These gene-specific rates of evolution, termed relative evolutionary rates (RER), reflect the amount of sequence divergence on a particular branch after correcting for non-specific factors affecting divergence on the branch such as time since speciation and mutation rate. Additionally, using a combination of statistical approaches including data transformation and weighted linear regression, RERconverge provides estimates of relative evolutionary rates that are robust to several factors introducing outliers in the dataset, such as the presence of distantly related species in the phylogeny (Partha et al. prep. To accelerate the computations underlying RER calculations, key functions are written in $\mathrm{C}++$ and integrated with the $\mathrm{R}$ code through the Rcpp package (Eddelbuettel and François, 2011).

\subsection{Flexible specification of binary and continuous trait evolution}

For binary traits, RERconverge provides multiple methods for users to specify which branches are in the "foreground", meaning those that display the convergent trait of interest. Users can directly supply foreground branch assignments by providing a phylogenetic tree with appropriate branch lengths or by interactively selecting branches on a phylogeny. Alternatively, users can specify which extant species are foreground and allow the software to infer which internal branches to consider foreground under a variety of scenarios. These include whether to consider only branches at a transition to a convergent phenotype as foreground or to also consider subsequent branches that preserve the phenotype as foreground, whether the foreground trait can be lost or only gained, and whether each foreground branch should be weighted equally.

In addition to supporting analysis of binary traits, RERconverge can perform correlation analysis between evolutionary rates and continuous traits Kowalczyk et al. prep). Trait evolution is modeled on a trait tree constructed 
from user-provided phenotype values for extant species provided by the user and ancestral nodes inferred through a phylogenetic model. By default, trait tree branch lengths represent change in the trait between a species and its ancestor, which removes phylogenetic dependence between branches. For completeness, other options allow branch lengths to be calculated as the average or terminal trait value along a branch to represent the state of a trait rather than its change.

\subsection{Genome-wide association between RER and traits, with correction for multiple testing}

RERconverge rapidly computes the association between genespecific relative evolutionary rates and the user-specified traits for large sets of genes. The program estimates the correlation between gene tree branch lengths and the values for traits along those branches using (by default) Kendall's Tau for binary traits and a Pearson linear correlation for continuous traits. Full gene lists are returned with correlation statistic values, the number of data points used for the correlations, and adjusted pvalues (FDR) derived using the Benjamini-Hochberg correction (Benjamini and Hochberg, 1995).

\subsection{Assessing gene set enrichment in results}

Further analysis of gene lists from correlation analyses can be performed using built-in RERconverge pathway enrichment functions. These functions perform a rank-based enrichment analysis by using a Wilcoxon Rank-Sum Test to detect distribution shifts between a subset of genes and all genes in annotated pathways. Enrichment results are returned with pathway names, enrichment statistics, Benjamini-Hochberg corrected p-values, and ranked pathway genes.

\section{Case study}

We demonstrate the features of RERconverge using gene trees derived from the coding sequences of 19,149 genes for 62 mammal species, along with foreground branches that represent lineages of the mammalian phylogeny whose members live predominantly in marine aquatic environments (Chikina et al. 2016 Meyer et al. 2018 Partha et al. prep. For this dataset, RERconverge ran in 1 hour and 18 minutes on RStudio with $\mathrm{R}$ version 3.3.0 installed on a computer running Windows 10 with $16 \mathrm{~GB}$ of RAM and an Intel Core i7-6500U $2.50 \mathrm{GHz}$ CPU. Top categories of functional enrichment included olfactory transduction $(\mathrm{FDR}=7.2 \mathrm{e}-90)$, GPCR signaling $(\mathrm{FDR}=1.4 \mathrm{e}-38)$, and immune functions $(\mathrm{FDR}=5.4 \mathrm{e}-7)$, as in Chikina et al. (2016). We show the RERconverge workflow and visualization of RERs for a top olfactory receptor, OR9Q2, in Figure 1.

\section{Summary}

RERconverge is an easy-to-implement method that can quickly test for associations between genes' relative evolutionary rates and traits of interest on a phylogeny. This enables researchers studying a wide variety of questions to generate lists of candidate genes associated with evolutionarily important traits and to explore through enrichment analyses the biological functions showing the most evidence of molecular change in association with these traits.

\section{Acknowledgments}

We thank Andreas Pfenning, Qingyuan Zhao, Ben Rubin, and Tim Sackton for feedback on earlier versions of this software, as well as all members of the Chikina, Clark and Kostka labs for helpful discussion. This project was supported by NIH R01 HG009299-01A1 to N.L.C. and M.C. A.K. was supported by NIH T32 training grant T32 EB009403 as part of the HHMINIBIB Interfaces Initiative.

\section{References}

Benjamini, Y. and Hochberg, Y. (1995). Controlling the False Discovery Rate: A Practical and Powerful Approach to Multiple Testing.

Chikina, M., Robinson, J. D., and Clark, N. L. (2016). Hundreds of Genes Experienced Convergent Shifts in Selective Pressure in Marine Mammals. Molecular biology and evolution, 33(9), 218292 .

Eddelbuettel, D. and François, R. (2011). Rcpp: Seamless R and C++ integration. Journal of Statistical Software, 40(8), 1-18. Feldman, C. R., Brodie, E. D., and Pfrender, M. E. (2012). Constraint shapes convergence in tetrodotoxin-resistant sodium channels of snakes. Proceedings of the National Academy of Sciences of the United States of America, 109(12), 4556-61.

Hiller, M., Schaar, B., Indjeian, V., Kingsley, D., Hagey, L.

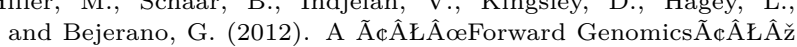
Approach Links Genotype to Phenotype using Independent Phenotypic Losses among Related Species. Technical Report 4.

Hu, Z., Sackton, T. B., Edwards, S. V., and Liu, J. S. (2018). Bayesian detection of convergent rate changes of conserved noncoding elements on phylogenetic trees. bioRxiv, page 260745 . Kowalczyk, A., Partha, R., Clark, N. L., and Chikina, M. (In prep) Kowalczyk, A., Partha, R., Clark, N. L., and Chikina, M. (In prep).
Control of cancer is a key functionality underlying evolution of extended lifespan in mammals.

Kronforst, M. R., Barsh, G. S., Kopp, A., Mallet, J., Monteiro A., Mullen, S. P., Protas, M., Rosenblum, E. B., Schneider, C. J., and Hoekstra, H. E. (2012). Unraveling the thread of nature's tapestry: the genetics of diversity and convergence in animal pigmentation. Pigment Cell \& \& Melanoma Research, $\mathbf{2 5}(4), 411-433$.

25(4), 411-433.
Lartillot, N. and Poujol, R. (2011). A phylogenetic model for investigating correlated evolution of substitution rates and continuous phenotypic characters. Molecular biology and evolution, 28(1), 729-44.

Li, Y., Liu, Z., Shi, P., and Zhang, J. (2010). The hearing gene Prestin unites echolocating bats and whales. Current biology $C B, \mathbf{2 0}(2), \mathrm{R} 55-6$.

Meyer, W. K., Jamison, J., Richter, R., Woods, S. E., Partha, R. Kowalczyk, A., Kronk, C., Chikina, M., Bonde, R. K., Crocker D. E., Gaspard, J., Lanyon, J. M., Marsillach, J., Furlong, C. E., and Clark, N. L. (2018). Ancient convergent losses of Paraoxonase 1 yield potential risks for modern marine mammals. Science (New York, N.Y.), 361(6402), 591-594.

Partha, R., Chauhan, B. K., Ferreira, Z., Robinson, J. D. Lathrop, K., Nischal, K. K., Chikina, M., and Clark, N. L. (2017). Subterranean mammals show convergent regression in ocular genes and enhancers, along with adaptation to tunneling. eLife, 6, e25884.

Partha, R., Kowalczyk, A., Clark, N. L., and Chikina, M. (In prep). Robust methods for detecting convergent shifts in evolutionary rates.

Prudent, X., Parra, G., Schwede, P., Roscito, J. G., and Hiller, M. (2016). Controlling for Phylogenetic Relatedness and Evolutionary Rates Improves the Discovery of Associations Between Species' Phenotypic and Genomic Differences. Molecular biology and evolution, $\mathbf{3 3}(8), 2135-50$.

$\mathrm{R}$ Core Team (2017). R: A Language and Environment for Statistical Computing. R Foundation for Statistical Computing, Vienna, Austria

Yang, Z. (2007). PAML 4: phylogenetic analysis by maximum likelihood. Molecular biology and evolution, 24(8), 1586-1591. 